\title{
Chronic total occlusions - a stiff challenge requiring a major breakthrough: is there light at the end of the tunnel?
}

\section{S Aziz, D R Ramsdale}

$\longrightarrow \mathrm{d}$ hronic total occlusion (CTO) remains one of the more difficult challenges for coronary interventionists. Innovations are essential if old, calcified, and long CTOs are to be successfully recanalised without acute complication and with a satisfactory short and long term outcome. Recent developments have included devices for breaking through the fibrous cap by blunt microdissection, an ultrasound device for "softening" resistant CTOs, and a device for differentiating between plaque and normal vessel wall to aid safe passage of a guidewire to the lumen beyond. This paper will review current practice and recent technological advances that are aimed at overcoming problematic CTOs.

\section{DEFINITION}

Although it is often impossible to know exactly when the occlusion occurred, a CTO is arbitrarily defined as a $>3$ month old, total obstruction of a coronary artery. A definition of $>1$ month is likely to result in higher reported success rates for a particular treatment strategy.

\section{PATHOLOGY}

CTOs consist of various degrees of fibro-atheromatous plaque and thrombus depending on the mechanism of occlusion and its duration (fig 1). A tough fibrous cap is often present at the proximal and distal margins of the CTO, with softer material in between. Endothelialised microchannels that traverse the occlusion increase the likelihood of passage with low profile hydrophilic guidewires. When the fibrous occlusion is long, densely organised and homogenous, and when microchannels are absent, guidewire passage is less successful and subintimal dissection likely.

\section{The clinical and technical problem}

Approximately $30 \%$ of all coronary angiograms in patients with coronary artery disease will show a CTO and its presence often excludes patients from treatment by percutaneous coronary intervention (PCI). ${ }^{1}$ CTOs are one of the commonest reasons for referral for coronary artery bypass surgery (CABG) and many are left untreated because of uncertainty regarding procedural success and long term benefit. ${ }^{2}$ The British Cardiovascular Intervention Society (BCIS) audit for 2002 showed that only $9 \%$ of all PCI procedures are for single vessel CTOs and this has changed little over the last decade $^{3}$-reflecting the anticipated low likelihood of success for this difficult subgroup.

The technical challenges include passage of a guidewire and balloon catheter across the CTO to enable successful PCI, and the maintenance of both short and long term patency.

\section{Rationale for opening CTOs}

The most common indication is to relieve angina pectoris or inducible myocardial ischaemia occurring as a result of the occluded vessel, as an alternative to CABG. Others include improvement of left ventricular (LV) function, the safe

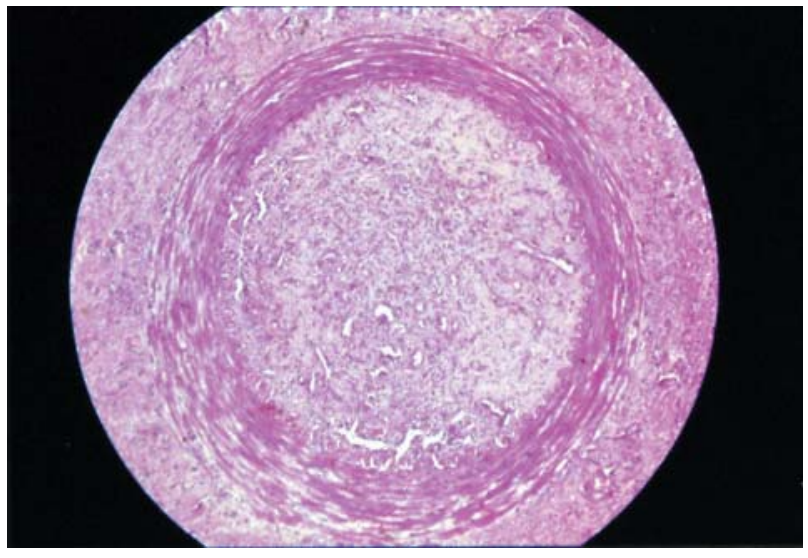

Figure 1 Microscopic section through a chronic total occlusion (CTO) with visible tiny microchannels. Courtesy of Dr John Gosney, Royal Liverpool University Hospital, Liverpool.

facilitation of multivessel PCI, and the improvement in long term survival.

\section{Predictors of success and failure}

Factors which predict a lower procedural success rate include length of occlusion $>15 \mathrm{~mm}$, duration of occlusion $>3$ months, presence of calcification in lesion and/or artery, and the presence of bridging collaterals. A "tapered-tip" occlusion is more favourable than a blunt "cut-off", as is the absence of side branches in close proximity to the occlusion. ${ }^{45}$ Most interventionists agree that there is a learning curve for CTO PCI, which affects success rates.

\section{Current $\mathrm{PCl}$ technique}

Before PCI, it is important to review the angiogram for favourable and unfavourable features. Multiple views of the lesion and its collaterals should be studied and an assessment made of the safety and risks of the procedure and the need for surgical back up. Previous angiograms showing the shape of the vessel when it was patent provide a valuable "road map". Demonstration of distal collaterals may help assess the occlusion length and direct guidewire passage during PCI. Simultaneous injection of the coronary artery supplying the collaterals using a catheter placed via the contralateral femoral artery may confirm that the guidewire is in the distal vessel beyond the CTO.

Abbreviations: $\mathrm{BCIS}$, British Cardiovascular Intervention Society; $C A B G$, coronary artery bypass graft surgery; $C T O$, chronic total occlusion; DES, drug eluting stents; LV, left ventricle; LVEF, left ventricular ejection fraction; MACE, major adverse cardiac event; MGC, micro guide catheter; $\mathrm{MI}$, myocardial infarction; OCR, optical coherence reflectometry; $\mathrm{PCl}$, percutaneous coronary intervention; PTCA, percutaneous transluminal coronary angioplasty; TLR, target lesion revascularisation 
The guide catheter should provide excellent back up support. Over-the-wire balloon catheters allow contrast injection into the distal coronary bed once the guidewire appears to be in the lumen beyond the occlusion. However, most operators have given up this advantage for rapid exchange devices and use contrast injection down the guide catheter to monitor progress.

\section{Choice of equipment}

Routine guidewires used to cross non-occlusive lesions do not have the tip stiffness or push sufficient to traverse the tough fibrous cap of CTOs. Specialised guidewires have a thicker core that gradually tapers towards the tip, increasing tip stiffness and support.

Moderately stiff Teflon coated guidewires such as the 0.014 inch diameter Intermediate or Standard guidewire (Guidant) may be the initial choice, especially if a "dimple" is evident. A low profile balloon catheter advanced close to the tip of the guidewire provides extra support for the wire as it is pushed into the occlusion.

The Shinobi guidewire (Cordis, Johnston \& Johnston) is specifically designed to cross CTOs and has a one piece core with a broad transition and increased stiffness, with a tip diameter of $0.014 \mathrm{inch}$. The distal $25 \mathrm{~cm}$ of the guidewire has a Teflon coating.

Some enhanced force Japanese guidewires also specifically designed for CTO are now distributed in Europe by Abbot Vascular devices. The Miracle guidewires (Asahi Intecc, Japan) have 0.014 inch tips and are available in several degrees of stiffness ( $3 \mathrm{~g} / 4.5 \mathrm{~g} / 6 \mathrm{~g} / \mathrm{l} 2 \mathrm{~g}$ ). The Conquest guidewire (Asahi Intecc) has a tip stiffness of $9 \mathrm{~g}$ and a distal diameter of 0.009 inch. Extreme care must be taken when using these stiff guidewires to cross CTOs, as they are more likely to create false channels, dissection and perforation.

Guidewires are traditionally coated with Teflon or silicone to provide a low friction surface. More recently, hydrophilic coated guidewires have been developed-for example, Conquest Pro (fig 2). These wires (table 1) have a polymer coating that becomes extremely slippery once moistened, which reduces thrombus adhesion and facilitates the advancement of the wire within the occlusion.

The Cross-IT XT guidewires (Guidant) have both a hydrophilic coating and a long tapered core that runs to the tip and provides extra support. The tip outer diameter is 0.010 inch which may help to traverse any microchannels while having the "push" of a 0.014 inch wire. The Cross-IT XT is manufactured in four grades from 100-400, with increasing torque transmission and support as flexibility decreases.

The new Magnum recanalisation wire (Biotronik, Bulach, Switzerland) has an olive tip with a smooth surface and
Table 1 Currently available hydrophilic coated guidewires suitable for crossing CTOs

\begin{tabular}{ll}
\hline Wire & Manufacturer \\
\hline${ }^{*}$ Hi-Torque Pilot & Guidant \\
†Cross-IT XT & Guidant \\
Whispher MS & Guidant \\
PT Graphix & Boston Scientific \\
Crosswire NT & Terumo \\
Galeo Hydro & Biotronik \\
Magnum & Biotronik \\
Conquest Pro & Asahi Intecc
\end{tabular}

${ }^{*}$ Available in three grades of increasing tip stiffness with $\mathrm{Hi}$ Torque Pilot 50, 150, and 200.

†Available in four grades of increasing tip stiffness with Cross-IT XT 100, 200, 300, and 400 .

edges (fig 3) for improved crossability. Its distal $30 \mathrm{~cm}$ has a hydrophilic coating and the wire can be extended to $325 \mathrm{~cm}$ using the Galeo extension wire. Its relatively atraumatic tip may reduce dissection and perforation and help avoid crossing stent interstices in stents occluded by fibrointimal hyperplasia. The effectiveness of the original Magnum wire has been reported in a large series of CTOs. ${ }^{6}$

If the distal lumen is reached, the lowest profile balloon catheter should then be advanced across the occlusion and the vessel reopened by percutaneous transluminal coronary angioplasty (PTCA) starting at the distal end of the occlusion and working proximally. A $1.5 \mathrm{~mm}$ balloon catheter with a single central marker is ideal and a short balloon $(10 \mathrm{~mm})$ may further improve the chance of crossing the occlusion. Further dilatation with a larger balloon may provide information about vessel size and distal disease before stent implantation. Drug eluting stents (DES) may prove to be most effective for maintaining long term patency with low in-stent restenosis rates.

In the event of a failure-to-cross with any guidewire, the Prima excimer laser wire (Spectranetics International) may be worth considering even though the total occlusion trial with angioplasty using the laser guidewire (TOTAL) failed to show any advantage over conventional guidewires for crossing CTOs.

If wire crossing is successful but the smallest balloon catheter will not cross, one option may be to ablate a channel using excimer laser atherectomy or rotational atherectomy using the Rotablator (Boston Scientific). For the latter it is necessary to exchange to a 0.009 inch Rotawire and this may mean loss of all that has been gained. Debulking before stenting may improve immediate angiographic success and

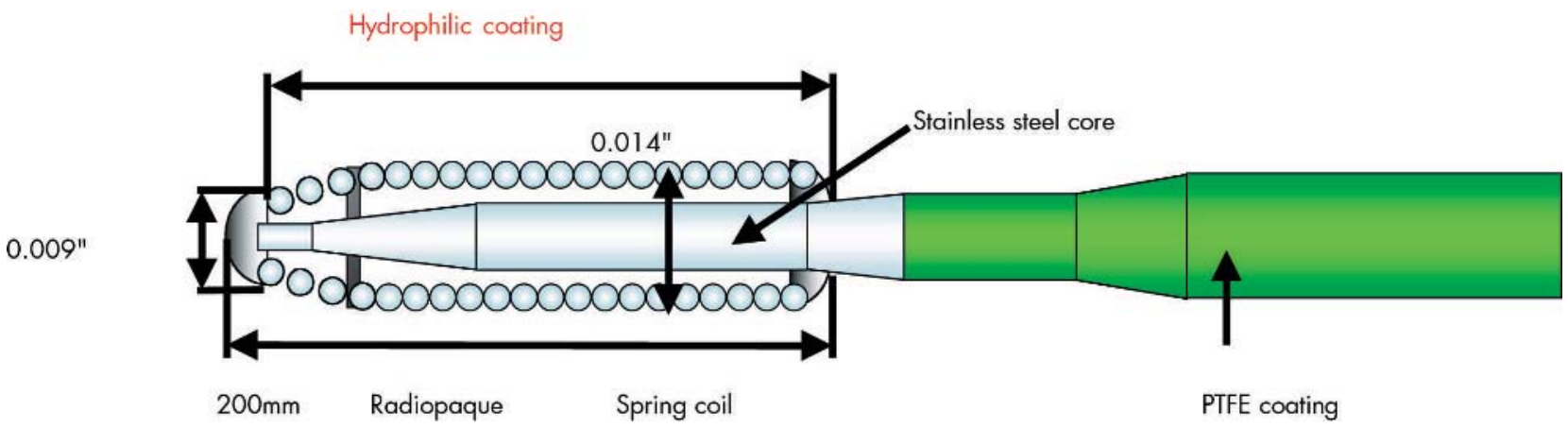

Figure 2 Conquest Pro guidewire incorporating both a distal tapered tip design with a diameter of 0.009 inch and a hydrophilic coating. A polytetrafluoroethylene (PTFE) coating is present on the proximal section of the guidewire. Courtesy of Asahi Intecc Co, European Office, Amsterdam, Netherlands. 

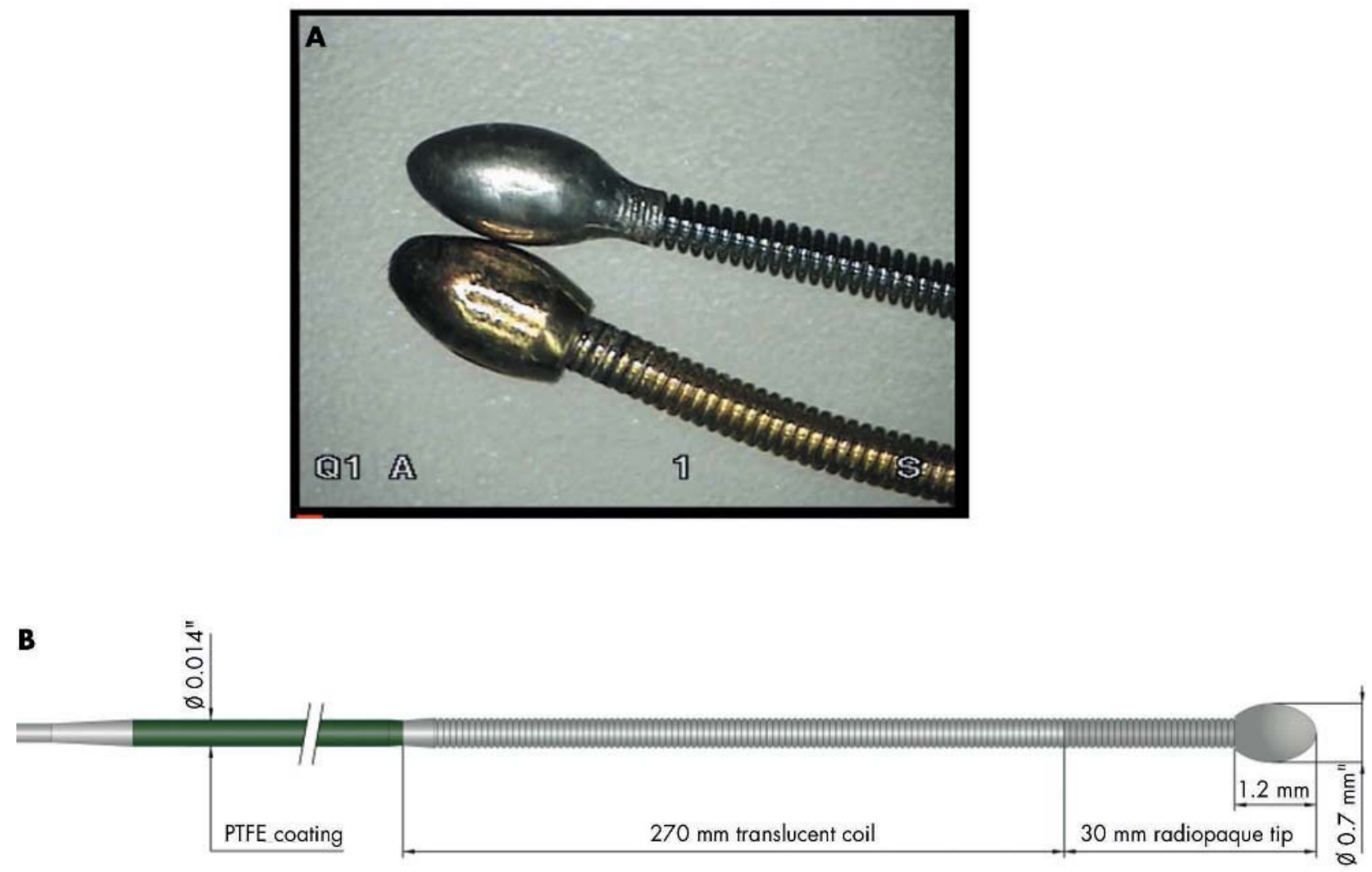

Figure 3 (A). The new 0.014 inch Magnum wire has a $0.7 \mathrm{~mm}$ diameter, $1.2 \mathrm{~mm}$ long radiopaque olive tip, which has a smoother surface and edges (top) than the original (bottom). (B) The distal $3 \mathrm{~cm}$ is radiopaque with a hydrophilic coating.

better stent deployment but does not appear to have any other benefit. ${ }^{8}$

\section{Complications}

The procedural complications include failure to cross with the guidewire or balloon, failure to dilate the lesion, failure to deploy a stent, and myocardial infarction (MI). Coronary artery dissection and/or perforation may lead to haemopericardium, cardiac tamponade, and death unless prompt pericardiocentesis and reversal of anticoagulation halts the demise. Emergency CABG surgery may be necessary.

Olivari et $a l^{9}$ reported an in-hospital major adverse cardiac event (MACE) rate of $5.1 \%$ when performing PCI for CTOs, which was largely due to non-Q wave MI in $4.25 \%$ of patients. Vessel perforation occurred in $2.1 \%$ of cases. Suero et $a l^{10}$ showed that the in-hospital MACE rates were similar for CTO and non-CTO lesions (3.8\% v 3.7\%, respectively).

\section{CLINICAL OUTCOMES}

\section{Acute recanalisation rates}

Acute success rates vary according to the definition of $>1$ or $>3$ months duration of occlusion, the morphology of the lesion and the coronary anatomy, the experience of the operator, the degree of persistence employed, and the type of equipment used. Recanalisation rates range between $45-80 \%$, with the highest success in short, recently occluded $(<1$ month), non-calcified lesions in non-tortuous vessels and in "functional" occlusions (fig 4). ${ }^{411}{ }^{12}$ BCIS reported a procedural success rate of $68 \%$ for CTOs in 2002 compared to $95 \%$ for non-occluded vessels. ${ }^{3}$

\section{Symptoms}

Successful recanalisation of a CTO in symptomatic patients improves angina pectoris. Berger et al reported $87 \%$ of patients to be asymptomatic at six months after successful PTCA of CTOs, ${ }^{13}$ and Ivanhoe et al reported that $69 \%$ were symptom-free at 36 months. ${ }^{14}$

\section{Left ventricular function}

In patients with angina or inducible ischaemia, recanalisation of CTOs has been shown to be associated with an improvement in LV ejection fraction (LVEF) from $62 \%$ to $67 \%$ $(\mathrm{p}<0.001)$-principally in regions supplied by the occluded artery. ${ }^{15}$

In patients with previous MI, improvement in LVEF (46.5\% to $49.7 \%, \mathrm{p}<0.05$ ) can be demonstrated in those with well developed collaterals but not in those without. However, the improvement is less than that seen in patients without a previous MI $(59.5 \%$ to $67.3 \%$, p < 0.001$)$. Such collaterals may be a marker for hibernating myocardium. ${ }^{16}$

\section{Late patency rates}

Six randomised trials have shown that stenting is superior to PTCA for CTOs with an improved acute angiographic appearance, less restenosis and reocclusion, and lower MACE and target lesion revascularisation (TLR) rates (fig 5). ${ }^{17-22}$

However, even with stenting, long term patency rates are suboptimal in long lesions. When the length of occlusion is $>50 \mathrm{~mm}$, angiographic restenosis with bare metal stenting 

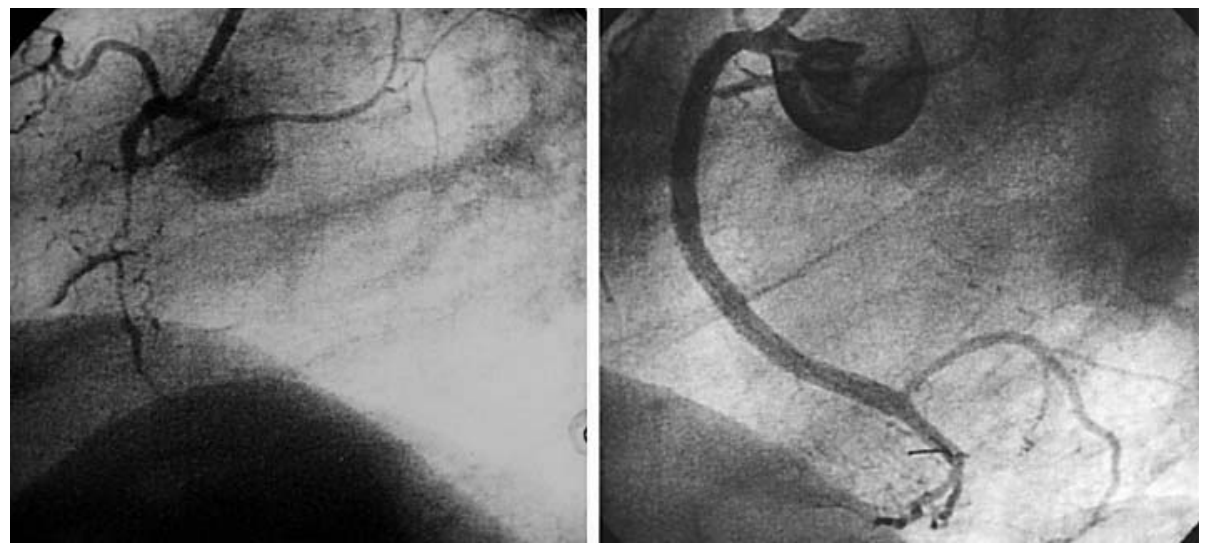

Figure 4 Angiogram of chronically occluded right coronary artery before and after $\mathrm{PCl}$ and stenting.

may be $>50 \%{ }^{23}$ Reocclusion is associated with a worse late outcome. ${ }^{24}$

In non-occlusive native coronary stenoses, DES have been shown to reduce the rates of angiographic restenosis compared to bare metal stents. ${ }^{25}$ Although the clinical trials have excluded patients with CTOs, registry data suggest that DES are likely to further reduce restenosis and reocclusion rates. ${ }^{26}$ A study of 33 patients with CTOs treated with sirolimus eluting stents has reported a six month angiographic binary restenosis rate of $9.1 \%$ with reocclusion in one patient. ${ }^{27}$ A prospective observational study, the ACROSS-Cypher study (approach to chronic occlusions with sirolimus stents) plans to recruit 250 patients with CTOs successfully treated with Cypher stents (Cordis/Johnson\&Johnson) to assess the effects on angiographic restenosis at six months, in-stent late loss, vessel patency and death, MI, and TLR. No studies are ongoing using the Taxus stent (Boston Scientific) in CTOs.

\section{Prognosis}

Long term survival benefit from CTO revascularisation has been documented in three observational clinical studies. In a single centre study with 2007 patients undergoing PCI for a CTO between 1980 and 1999, procedural success was achieved in $72.3 \%$ of cases. ${ }^{10}$ Only $7 \%$ of cases received a stent. The 10 year survival rate for patients with successful CTO treatment was higher than those with failed recanalisation $(73.5 \% \vee 65.1 \%, p=0.001)$ which proved an independent predictor of mortality.

The British Columbia cardiac registry included 963 patients undergoing a PCI for a CTO between 1993 and 2000. After
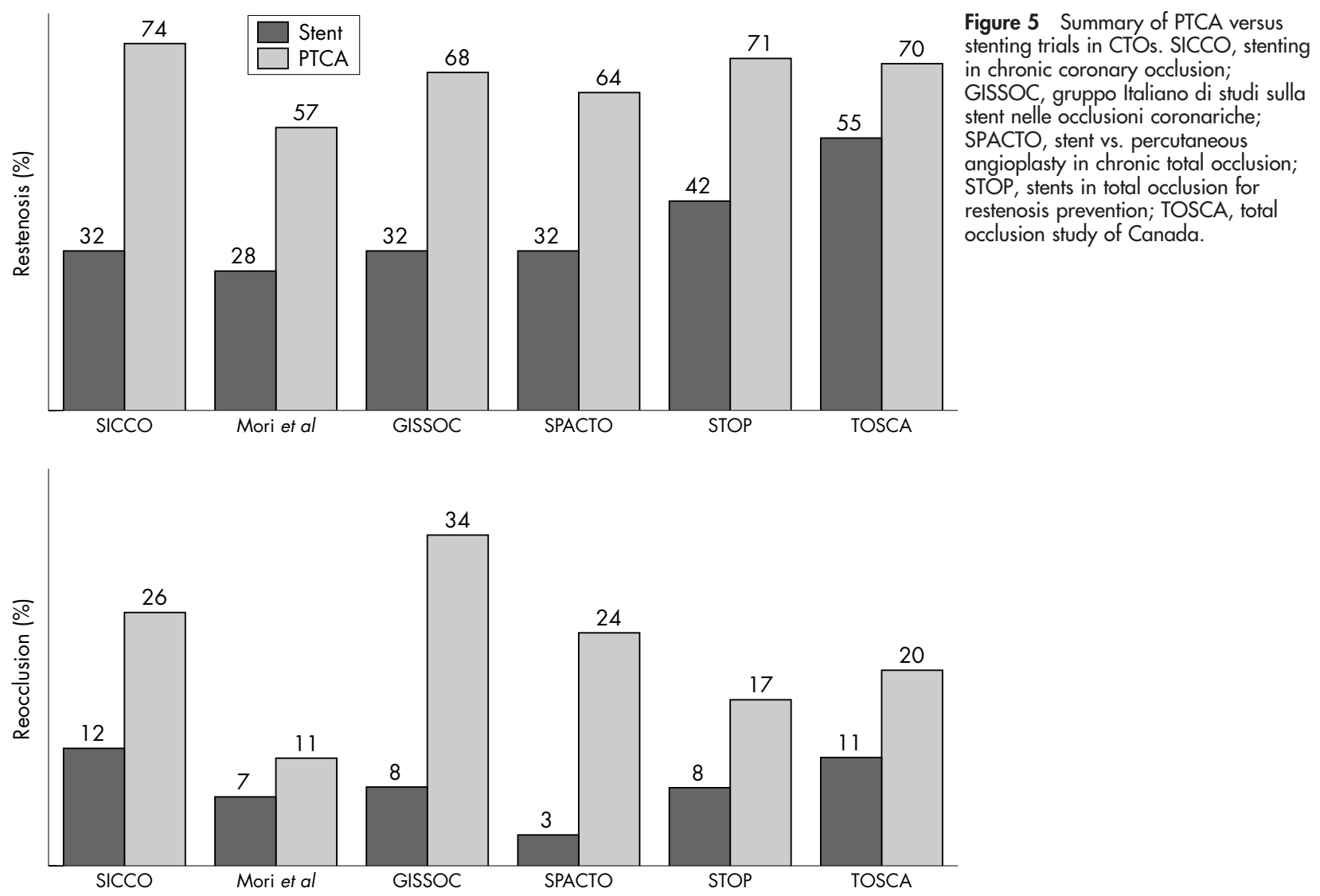
one year, survival was $98.2 \%$ in those successfully revascularised versus $94.8 \%$ in the failed group $(p<0.001){ }^{28}$

A prospective multicentre Italian observational study reported the success rate and clinical outcome of PCI for 390 CTOs (aged $>30$ days). ${ }^{9}$ The procedural success rate was $73.3 \%$. Stenting was used in $90 \%$ of all successfully recanalised CTOs. At 12 months, patients with a successful procedure had a lower incidence of cardiac death or MI $(1.05 \% \vee 7.23 \% ; p=0.005)$, a reduced need for CABG surgery $(2.45 \% v 15.7 \% ; \mathrm{p}<0.0001)$, and were more likely to be free of angina $(88.7 \% v 75 \% ; \mathrm{p}=0.008)$ than those with a failed procedure. CTO length of $>15 \mathrm{~mm}$ or not measurable, moderate to severe calcification, occlusion for $>180$ days, and multivessel disease were significant predictors of PCI failure.

\section{New devices}

Several new devices are under development and clinical evaluation in the hope of increasing procedural success and reducing complications.

\section{Frontrunner-XR}

The Frontrunner catheter (LuMend Inc, Redwood City, California) is a $135 \mathrm{~cm}$ long catheter with distal bioptomelike jaws, which can be opened and closed manually to a diameter of $3.0-4.2 \mathrm{~mm}$. It uses controlled blunt microdissection to create a channel through the CTO. The dissection planes are limited to the fibrocalcific plaque, which is more rigid and less stretchable than the vessel adventitia-reducing the risk of vessel perforation..$^{29}{ }^{30}$ The latest Frontrunner X39 has a hinged distal tip that can be angled to $25^{\circ}$ and $36^{\circ}$, which has improved the ability to navigate tortuous vessels (fig 6). It can be used through a 6 French guide catheter and is supported by a 4 French micro guide catheter (MGC). Once across the CTO, the MGC can be advanced into the distal lumen to function as a conduit for wire exchange.
A registry of 119 cases with CTOs in native coronary arteries uncrossable with conventional guidewires showed successful placement of the guidewire distal to the occlusion in $56 \%$ of cases using the original Frontrunner. After 342 cases, reported complications were infrequent with a $2 \%$ perforation rate and no device related deaths. ${ }^{31}$ Lesion success rates with the latest X39 device may be improved, although clinical experience is limited and further registry data are being collected. The Frontrunner device is not available for use in Europe.

\section{Therapeutic ultrasound}

The Crosser system (FlowCardia Inc, California) uses high frequency mechanical vibrations to facilitate the crossing of CTOs by changing the structure and hardness of plaque. It consists of a catheter that is connected to an alternating current power supply via a transducer. The tip of the catheter vibrates at $20 \mathrm{MHz}$ and causes mechanical disruption which aids the passage of a guidewire across a CTO. The catheter tip has an olive shape, a hydrophilic coating, and is $1.1 \mathrm{~mm}$ in diameter, compatible with a 6 French guide catheter and a 0.014 inch guidewire. It is available in both rapid exchange and over-the-wire versions. Initial registry data from 25 patients with CTOs resistant to the passage of conventional guidewires has reported a $75 \%$ success rate in crossing the occlusion with the Crosser system. ${ }^{32}$

\section{SafeCross}

The SafeCross device (Intraluminal Therapeutics Inc) has a 0.014 inch optical guidewire (Intraluminal wire) that emits near infra-red light (10-30 u resolution). It is placed through a support catheter which helps control guidewire advancement and the guidewire has either a straight or angled tip. Optical coherence reflectometry (OCR) uses the low coherence light transmitted from the 0.007 inch optical fibre incorporated into the tip of the guidewire to reflect from the tissue ahead. Depending on the absorption and scatter pattern present in the reflected signal, the detector can
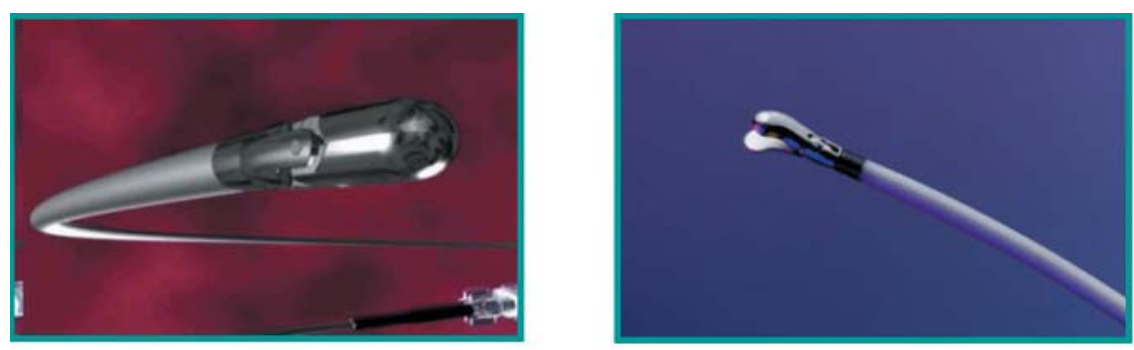

Figure 6 Frontrunner-XR catheter. The straight and hinged distal tip versions with bioptome-like jaws are shown. Courtesy of LuMend Inc, Redwood City, California.
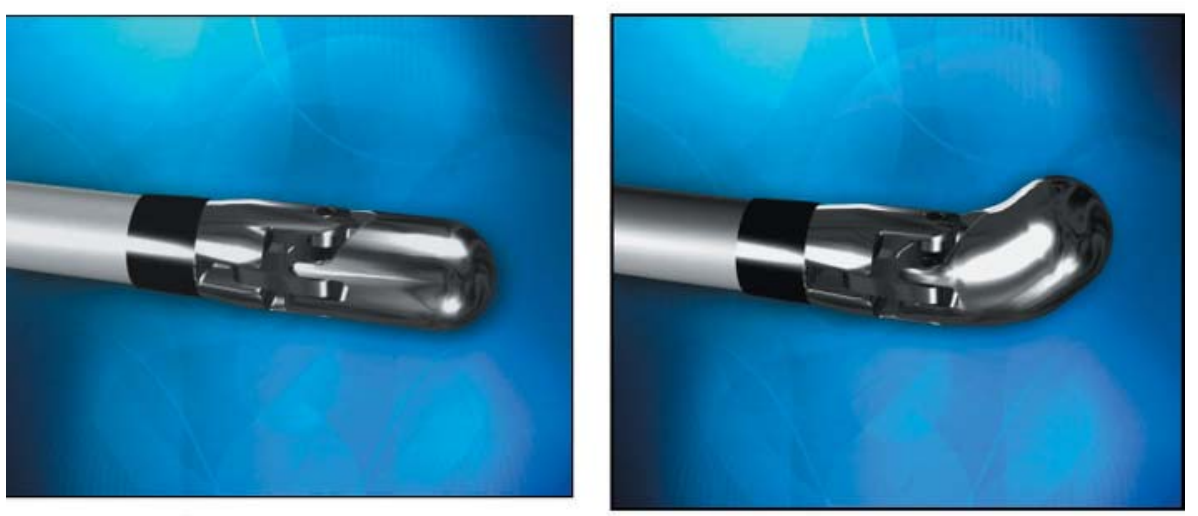

$0^{\circ}$ Angle

$36^{\circ}$ Angle 


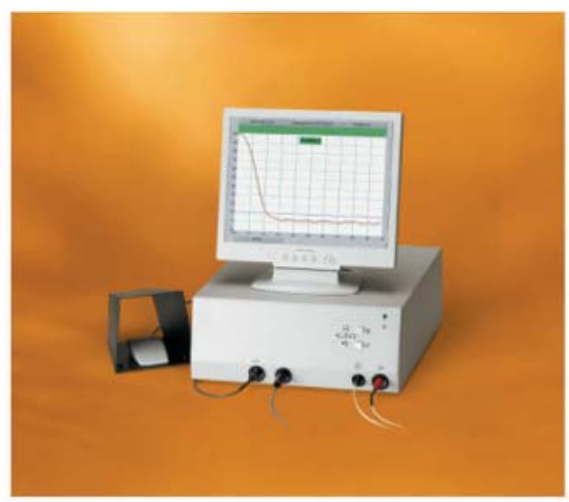

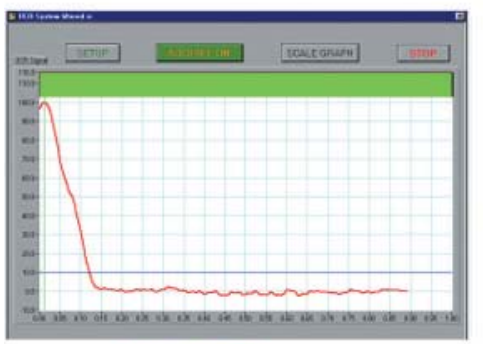

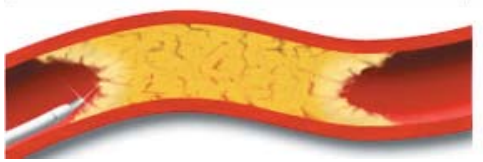

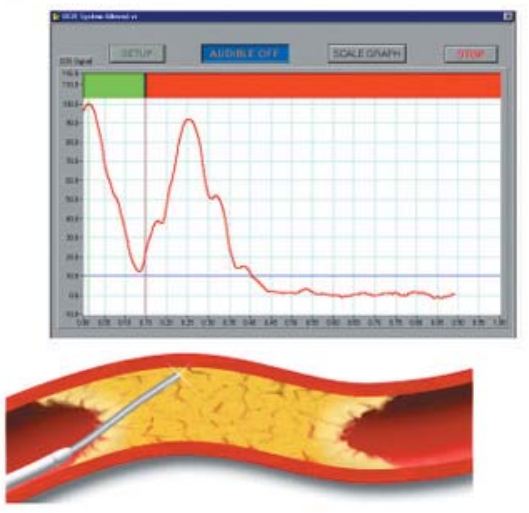

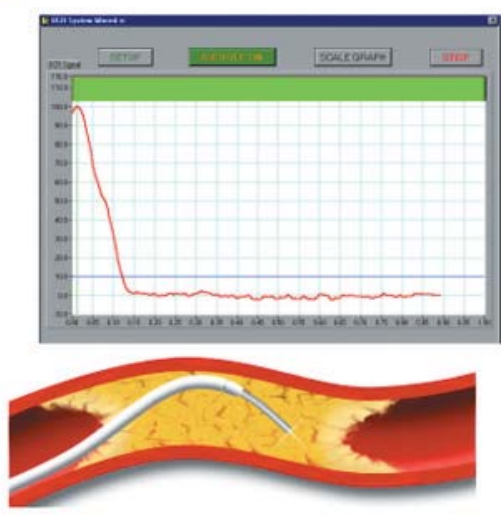

Figure 7 Use of optical coherence reflectometry to help distinguish between arterial wall and plaque with the SafeCross system. Courtesy of Intraluminal Therapeutics Inc, Carlsbad, California.

differentiate between plaque and normal artery wall. This information is displayed as a waveform on a screen, which enables the operator to determine the location of the guidewire within the occlusion (fig 7). By avoiding the vessel wall, the risk of perforation or dissection is reduced. Radiofrequency energy ( $100 \mathrm{~ms}$ pulses; $250-500 \mathrm{kHz}$ ) is emitted from the tip of the wire that enables it to traverse the tough fibrous cap of refractory CTOs.

In a registry of 32 patients whose CTO could not be crossed with a conventional guidewire, the device achieved recanalisation in $81 \%$ of cases without device complication. ${ }^{33}$ However, two further small studies report success in only $52-60 \%$ of such cases. ${ }^{34} 35$ Limited steerability within the lesion remains a problem. Interchange between conventional guidewires and SafeCross was necessary in hard lesions. A 440 patient prospective randomised trial GREAT (guided radiofrequency energy ablation of total occlusions) is currently underway to compare the effectiveness and safety of SafeCross compared to conventional wires.

\section{Plaque digestion}

Since most occlusive plaques consist mainly of collagen, experimental procedures in animals using a local infusion of collagenase onto CTOs have been performed and appears to aid successful crossing with a guidewire. ${ }^{36}$ However, whether this will ever be clinically useful remains uncertain.

\section{CONCLUSIONS}

Improved technique, specialised guidewires, and stenting have improved the success rates of PCI in CTOs, but innovations are required to deal with the more difficult subset of CTOs such as old, long, and calcified lesions with unfavourable anatomy. The novel devices described above now need to be formally tested to assess their true worth.

\section{Authors' affiliations}

S Aziz, D R Ramsdale, The Cardiothoracic Centre, Liverpool, UK

Correspondence to: Dr David R Ramsdale, The Cardiothoracic Centre Liverpool, Thomas Drive, Liverpool. L14 3PE, UK; David.Ramsdale@ctc. nhs.uk

\section{REFERENCES}

1 Bourassa MG, Roubin GS, Detre KM, et al. Bypass angioplasty revascularization investigation: patient screening, selection, and recruitment. Am J Cardiol 1995;75(9):3C-8C.

2 Delacretaz E, Meier B. Therapeutic strategy with total coronary artery occlusions. Am J Cardiol 1997;79:185-7.

3 BCIS. British Cardiovascular Intervention Society audit data 2002: www.bcis.org.uk.

4 Puma JA, Sketch MH Jr, Tcheng JE, et al. Percutaneous revascularization of chronic coronary occlusions: an overview. J Am Coll Cardiol 1995;26:1-11.

5 Noguchi T, Miyazaki MDS, Morii I, et al. Percutaneous transluminal coronary angioplasty of chronic total occlusions: determinants of primary success and long term clinical outcome. Catheter Cardiovasc Interv 2000;49:258-64.

6 Allemann Y, Kaufmann U, Meyer B, et al. Magnum wire for percutaneous transluminal coronary angioplasty in 800 total chronic occlusions. Am J Cardiol 1997;80:634-7.

7 Serruys PW, Hamburger JN, Koolen JJ, et al. Total occlusion trial with angioplasty by using laser guidewire. The TOTAL trial. Eur Heart $J$ 2000;21:1797-805.

8 Gruberg L, Mehran R, Dangas G, et al. Effect of plaque debulking and stenting on short and long-term outcomes after revascularization of chronic total occlusions. J Am Coll Cardiol 2000;35:151-6.

9 Olivari Z, Rubartelli P, Piscione F, et al. Immediate results and one-year clinical outcome after percutaneous coronary interventions in chronic total occlusion: data from a multicentre, prospective, observational study (TOASTGISE). J Am Coll Cardiol 2003;41:1672-8.

10 Suero JA, Marso SP, Jones PG, et al. Procedural outcomes and long-term survival among patients undergoing percutaneous coronary intervention of a chronic total occlusion in native coronary arteries: a 20 -year experience. J Am Coll Cardiol 2001;38:409-14.

11 Serruys PW, Umans V, Hendrickx GR, et al. Elective PTCA of totally occluded coronary arteries not associated with acute myocardial infarction: short-term and long-term results. Eur Heart J 1985;6:2-12.

12 Safian RD, McCabe CH, Sipperly ME, et al. Initial success and long-term follow-up of percutaneous transluminal coronary angioplasty in chronic total occlusions versus conventional stenoses. Am J Cardiol 1988;61:23G-8G. 
13 Berger PB, Holmes DR Jr, Ohman EM, et al. Restenosis, reocclusion and adverse cardiovascular events after successful balloon angioplasty of occluded versus nonoccluded coronary arteries. Results from the multicentre American research trial with cilazapril after angioplasty to prevent transluminal coronary obstruction and restenosis (MARCATOR). J Am Coll Cardiol 1996;27:1-7.

14 Ivanhoe RJ, Weintraub WS, Douglas JS, et al. Percutaneous transluminal coronary angioplasty of chronic total occlusions: primary success, restenosis and long-term clinical follow-up. Circulation 1992;85:106-15.

15 Sirnes PA, Myreng Y, Molstad P, et al. Improvement in left ventricular ejection fraction and wall motion after successful recanalization of chronic coronary occlusions. Eur Heart J 1998;19:273-81.

16 Chung CM, Nakamura S, Tanaka K, et al. Effect of recanalization of chronic total occlusions on global and regional left ventricular function in patients with or without previous myocardial infarction. Catheter Cardiovasc Interv 2003;60:368-74

17 Rubartelli P, Niccoli L, Verna E, et al. Stent implantation versus balloon angioplasty in chronic coronary occlusions: results from the GISSOC trial. Gruppo Italiano di Studio sullo Stent nelle Occlusioni Coronariche. J Am Coll Cardiol 1998;32:90-6.

18 Sirnes PA, Golf S, Myreng Y, et al. Stenting in chronic coronary occlusion (SICCO): a randomised, controlled trial of adding stent implantation after successful angioplasty. J Am Coll Cardiol 1996;28:1444-51.

19 Buller CE, Dzavik V, Carere RG, et al. Primary stenting versus balloon angioplasty in occluded coronary arteries: the total occlusion study of Canada (TOSCA). Circulation 1999; 100:236-42.

20 Hoher M, Wohrle J, Grebe OC, et al. A randomised trial of elective stenting after balloon recanalization of chronic total occlusions. J Am Coll Cardiol 1999:34:722-9.

21 Mori $M$, Kurogane $H$, Hayashi T, et al. Comparison of results of intracoronary implantation of the Palmaz-Schatz stent with conventional balloon angioplasty in chronic total coronary arterial occlusion. Am J Cardiol 1996;78:985-9.

22 Lotan C, Rozenman Y, Hendler A, et al. Stents in total occlusion for restenosis prevention. The multicentre randomised STOP study. The Israeli working group for interventional cardiology. Eur Heart J 2002;21:1960-6.

23 Sheiban I, Moretti C, Kumar P, et al. Immediate and medium-term outcomes following the treatment of very long $(\geqslant 50 \mathrm{~mm})$ chronic total coronary artery occlusions. J Invasive Cardiol 2004; 16:5-9.
24 Buller CE, Teo KK, Carere RG, et al. Three year clinical outcomes from the total occlusion study of Canada (TOSCA). Circulation 2000; 102:II-1885.

25 Moses JW, Leon MB, Popma JJ, et al. for the SIRIUS Investigators. Sirolimuseluting stents versus standard stents in patients with stenosis in a native coronary artery. N Engl J Med 2003;349:1315-23.

26 Cioppa A, Ambrosini V, Battaglia ML, et al. Recanalization of chronic total occlusion with sirolimus-eluting stent: single centre experience. Am J Cardiol 2003;92(suppl 6A):66L.

27 Hoye A, Tanabe K, Lemos PA, et al. Significant reduction in restenosis after the use of sirolimus-eluting stents in the treatment of chronic total occlusions. J Am Coll Cardiol 2004;43:1954-8.

28 Ramanathan K, Gao M, Nogareda GJ, et al. Successful percutaneous recanalization of a non-acute occluded coronary artery predicts clinical outcomes and survival. Circulation 2001;104:11-415.

29 Simonton SA. Chronic total occlusions: a new frontier. J Invasive Cardiol 2004;16(suppl B):1-2

30 Tadros T. Successful revascularisation of a long chronic total occlusion of the right coronary artery utilising the Frontrunner X39 catheter system. J Invasive Cardiol 2003;15(11):3.

31 Selmon MR, Daniel MA. Catheter assisted recanalization of chronic total occlusions in the coronary vasculature. Cardiology International 2003;4:79-82

32 Gabor Sütsch. The FlowCardia Crosser ${ }^{\mathrm{TM}}$ system for the recanalization of chronic total occlusions. EuroPCR Paris May 27, 2004.

33 Braden GA. Clinical experience in crossing total occlusions with the SafeCross system. Am J Cardiol 2003;92(uppl 6A):66L.

$34 \mathrm{Ng} \mathrm{W}$, Chen WH, Lee PY, et al. Initial experience and safety in the treatment of chronic total coronary occlusions with a new optical coherent reflectometry-guided radiofrequency ablation guidewire. Am J Cardiol 2003;92:732-4.

35 Hoye A, Cummins P, Onderwater E, et al. Improved rate of chronic total occlusion recanalisation utilising the Safe-Cross ${ }^{T M}$ system: a wire with the capacity of radiofrequency ablation that is guided by optical coherence reflectometry. Heart 2004;90(suppl II):A16.

36 Strauss BH, Goldman L, Qiang B, et al. Collagenase plaque digestion for facilitating guidewire crossing in chronic total occlusions. Circulation 2003;108:1259-62. 